\title{
ROMANIAN
}

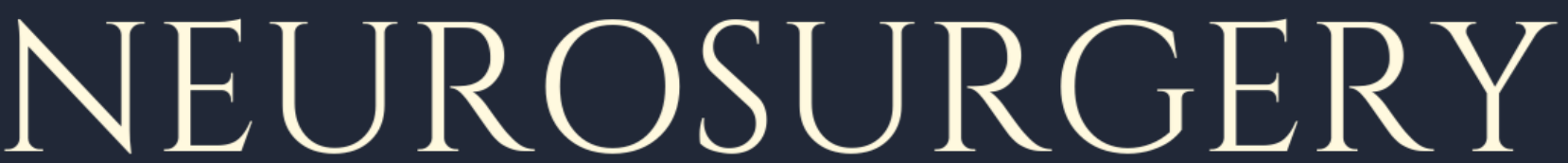

\author{
Vol. XXXIV | No. $1 \quad$ March 2020
}

\section{Efficacy of c-arm scopy-guided erector spinae plane block (ESPB) in postoperative pain control and reduction of opioid side effects in spinal instrumentation surgery}

\author{
Yasar Karatas, Fatih Keskin, \\ Mehmet Fatih Erdi, Bulent Kaya, \\ Ibrahim Kosemen, \\ Mehmet Kenan, Erdal Kalkan
}




\section{Efficacy of c-arm scopy-guided erector spinae plane block (ESPB) in postoperative pain control and reduction of opioid side effects in spinal instrumentation surgery}

\author{
Yasar Karatas ${ }^{1}$, Fatih Keskin ${ }^{2}$, Mehmet Fatih \\ Erdi $^{2}$, Bulent Kaya ${ }^{1}$, Ibrahim Kosemen ${ }^{1}$, Mehmet \\ Kenan², Erdal Kalkan ${ }^{1}$ \\ 1 Medova Hospital, Konya, TURKEY \\ 2 Necmettin Erbakan University, Meram Medical Faculty, Department \\ of Neurosurgery, Konya, TURKEY
}

\begin{abstract}
Background: Spinal instrumentation surgery causes significant pain in patients. As a result, patients are exposed to excessive opioid use and the associated side effects, as well as prolonged hospital stay, resulting in economic burden. Local anaesthetics can help both reduce postoperative pain and minimize the side effects associated with systemically administered opioid analgesics.

Objective: The aim of this retrospective study was to investigate the effect of erector spinae plane block on analgesia in spinal instrumentation surgery and to reduce the side effects of excessive opioid use.

Materials and Methods: We reported a retrospective study. Thirty patients, who underwent spinal instrumentation surgery from 2017 to 2018, were chosen from the hospital records. We performed spinal instrumentation and decompression by laminectomy to all patients under general anaesthesia. While 15 of these patients underwent erector spinae plane block, these patients received patient-controlled analgesia postoperative period. The other 15 patients received only patientcontrolled analgesia postoperative period. We analyzed patients' data for differences in preoperative and postoperative visual analogue scores, nausea vomiting scores, constipation life quality scale, patient-controlled analgesia shot count and mean opioid consumption of patients.
\end{abstract}

Results: The data of 30 patients undergoing lumbar spinal instrumentation surgery were retrospectively analyzed. There was no significant difference in the age, preoperative VAS, preoperative ODI and sex between the two groups $(p>0,05)$. In addition, there were statistically significant differences in postoperative VAS, postoperative ODI, Nausea Vomiting Score, Constipation Life Quality Score (CLQS), petidin consumption and PCA shot count $(p<0.05)$. In all variables with significant differences, the values in the block group were lower than the non-block group.

Conclusion: ESPB provides effective analgesia and reduces side effects due to excessive opioid usage.

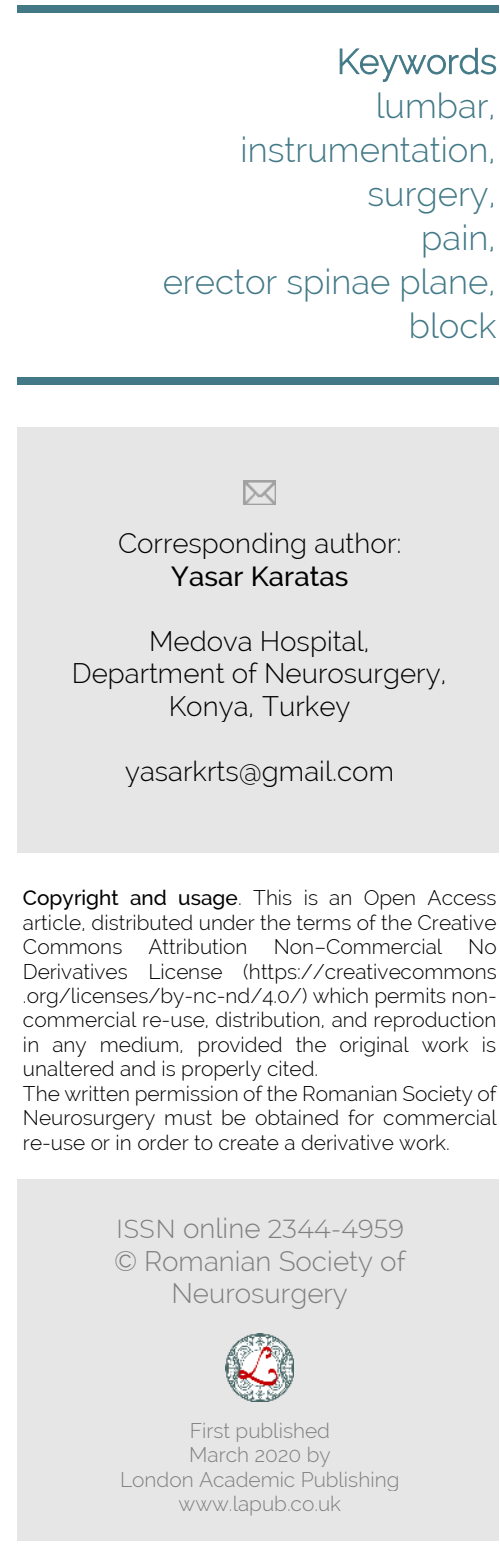

lumbar.

surgery,

pain,

block article distributed under the terms of the Creative Commons Attribution Non-Commercial No Derivatives License (https://creativecommons commercial re-use distribution, and reproduction in any medium, provided the original work is unaltered and is properly cited

The written permission of the Romanian Society of Neurosurgery must be obtained for commercial re-use or in order to create a derivative work

ISSN online 2344-4959 Romanian Society of Neurosurgery ww lapubcouk 


\section{INTRODUCTION}

Open lumbar spine instrumentation surgery causes significant soft tissue damage because of large dissection and stripping the muscles. As a result, patients complain of nociceptive, neuropathic and inflammatory pain. [1]. In recent years, pain control after spinal surgery has been targeted with minimal opiate use. The use of gabapentinoids, acetaminophen, neural blockages and long-term local anesthetics is supported by scientific evidence $[2,3]$. Erector spinae plane block (ESPB) was defined for thoracic and abdominal pain previously $[4,5]$. There are various articles about postoperative pain control of erector spinae plane block. However, for the first time, we present a study showing that the amount of excessive opioid use is reduced and the associated side effects are reduced.

\section{MATERIALS AND METHODS}

We reported a retrospective study. This retrospective study included the data of thirty patients, who underwent spinal instrumentation surgery from 2017 to 2018. Preoperative and postoperative data such as age, sex, VAS, ODI, constipation quality life score, nausea vomiting score, petidine use, number of pca shot were collected. Each patient's outcome was noted by the same surgeon. Postoperative VAS, CLQS, nausea vomiting score measured in postoperative day 1 and postoperative ODI measured in postoperative day 30.

\section{Surgery and postoperative protocol}

The same surgeon performed all operations. Instrumentation and decompression by laminectomies were performed for all patient through a midline incision followed by stripping of paraspinal musculature. The patients underwent the same level surgical procedure. The participants had access to intravenous patient-controlled analgesia that comprised $1 \mathrm{mg} / \mathrm{h}$ continuous pethidine administration and $20 \mathrm{mg}$ pethidine bolus infusion when requested by the participants. The bolus was permitted three times in a hour for all participants.

\section{Erector spinae plane block technique}

C-arm-scopy guided erector spinae plane block was performed just before surgıcal incision by the same surgeon. ESPB was made bilaterally at the level of instrumentation and decompression. After the patient was placed in prone position and sterile isolation was achieved, transverse process was determined by scopy. With a 20-G needle, the bottom of the transverse process was reached. The bilateral ESPB were performed by injecting $5 \%$ bupivacaine ( $20 \mathrm{~mL}$ into each side) for each level into the deep surface of the erector spinae muscle and the transverse processes of the lumbar vertebrae

\section{Statistical analysis}

Statistical analysis of the study was performed in $\mathrm{R}$ 3.1.2 package program. Descriptive measures of the quantitative variables in the study are shown with their median, minimum and maximum values. The suitability of the variables to normal distribution was examined by Shapiro Wilk test. Mann Whitney U test was used to compare two independent variables. Intragroup changes of variables were examined by Wilcoxon test. Fisher exact chi-square test was used for group comparison of qualitative variables. In all statistical analyzes in the study, $p$ values less than 0.05 were considered statistically significant.

\section{RESULTS}

There was no statistically significant difference between the groups in terms of age, preop VAS and preop ODI ( $p>0.05)$. In addition, there were statistically significant differences in postop VAS, postop ODI, Nausea Vomiting Score, CLQS, petidine consumption and PCA Shot Count ( $p<0.05$ ). In all variables with significant differences, the values in the non-block group were higher than the block group (Table 1). The change in VAS and ODI values in the block group was statistically significant ( $p$ $<0.001)$. Similarly, the change in VAS and ODI values were significant in the non-block group ( $p<0.001)$. Both VAS and ODI variables were significantly reduced in both groups (Figure 1). On the other hand, the mean decrease in VAS values in the block group was $79.0 \%$, whereas the mean decrease in VAS values in the non-block group was $67.1 \%$ and there was a statistically significant difference between the groups in terms of VAS values ( $p<0.001)$. In other words, there has been much more decline in the block group. (Figure 2) The mean decrease in ODI values in the block group was $55.4 \%$ and the mean decrease in ODI values in the non-block group was $48.3 \%$ and there was no statistically significant difference between the groups in terms of the decrease in ODI values ( $p=0.202)$. Decreases in ODI values were similar in both groups (Figure 3 ). There 
was no statistically significant difference between the groups in terms of gender $(p=1,000)$. In both groups, the ratio of men and women is $80 \%$ to $20 \%$ (Table 2 ).

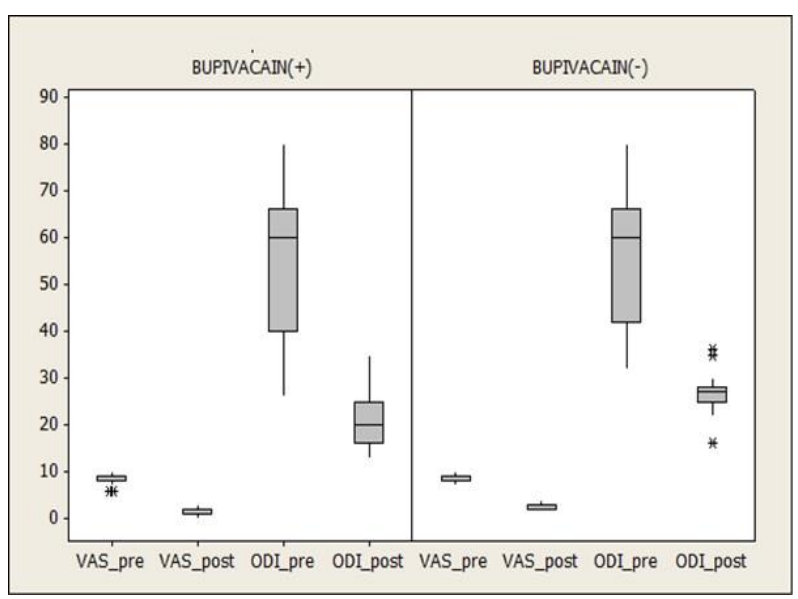

Figure 1. Both preoperative and postoperative VAS and ODI variables in the groups.

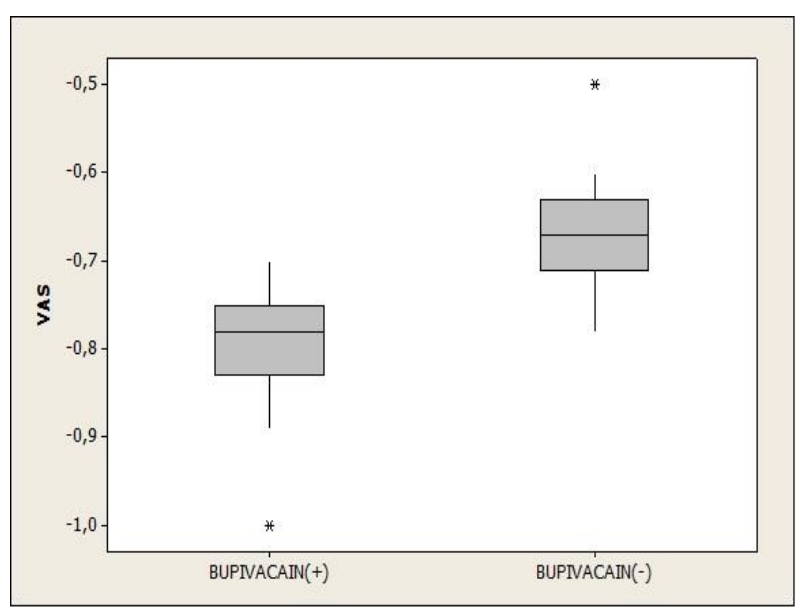

Figure 2. The mean decrease in VAS values in the groups.

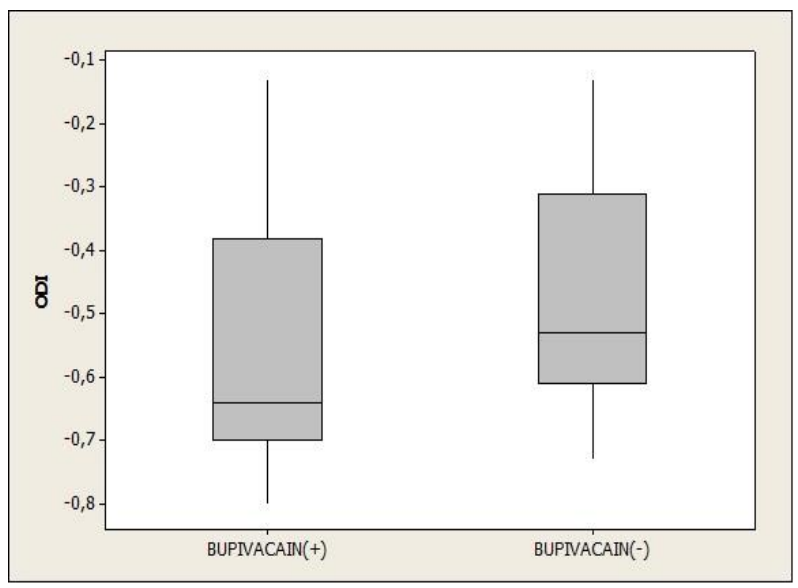

Figure 3. The mean decrease in ODI values in the groups.
Table 1. Mean age of groups, preoperative VAS, postoperative VAS, preoperative ODI, postoperative ODI, Nausea Vomiting Score, CLQS, petidine consumption and PCA Shot Count are shown in table.

\begin{tabular}{|c|c|c|c|}
\hline & ESPB WITH & ESPB & $p$ \\
\hline & \multirow[t]{2}{*}{ BUPIVACAIN } & WITHOUT & \\
\hline & & BUPIVACAIN & \\
\hline \multirow{2}{*}{ Age } & $61,13 \pm 18,28$ & $64,20 \pm 13,72$ & 0,870 \\
\hline & $66(14-81)$ & $69(38-85)$ & \\
\hline \multirow{2}{*}{ VAS preop } & $8,20 \pm 1,21$ & $8,73 \pm 0,88$ & 0,250 \\
\hline & $8(6-10)$ & $9(7-10)$ & \\
\hline \multirow{2}{*}{ VAS postop } & $1,73 \pm 0,70$ & $2,87 \pm 0,64$ & $<0,001$ \\
\hline & $2(0-3)$ & $3(2-4)$ & \\
\hline \multirow{2}{*}{ ODI preop } & $54,47 \pm 14,70$ & $55,53 \pm 14,03$ & 0,806 \\
\hline & $60(26-80)$ & $60(32-80)$ & \\
\hline \multirow{2}{*}{ ODI postop } & $22,13 \pm 6,83$ & $26,80 \pm 4,81$ & 0,023 \\
\hline & $20(13-35)$ & $27(16-36)$ & \\
\hline Nausea & $1,27 \pm 1,22$ & $3,07 \pm 1,71$ & 0,002 \\
\hline Vomiting & $1(0-4)$ & $3(1-8)$ & \\
\hline \multicolumn{4}{|l|}{ Score } \\
\hline \multirow{2}{*}{ CLQS } & $14,47 \pm 7,76$ & $26,93 \pm 8,67$ & $<0,001$ \\
\hline & $15(4-30)$ & $27(16-42)$ & \\
\hline \multirow{2}{*}{$\begin{array}{l}\text { Petidin } \\
\text { Comsumption }\end{array}$} & $42,53 \pm 22,96$ & $146,25 \pm 29,83$ & $<0,001$ \\
\hline & $35(12-91,6)$ & $149,3(85-200)$ & \\
\hline PCA Shot & $1,80 \pm 1,37$ & $7,40 \pm 1,60$ & $<0,001$ \\
\hline Count & $2(0-5)$ & $8(5-10)$ & \\
\hline
\end{tabular}

\#Mann Whitney U test

Table 2. The ratio of men and women in the groups. \#Mann Whitney $\mathrm{U}$ test

\begin{tabular}{llll}
\hline & $\begin{array}{l}\text { ESPB } \\
\text { BUPIVACAIN }\end{array}$ & $\begin{array}{l}\text { ESPB WITHOUT } \\
\text { BUPIVACAIN }\end{array}$ & $\mathrm{p}$ \\
\hline Male & $3(\% 20,0)$ & $3(\% 20,0)$ & \multirow{2}{*}{1,000} \\
Female & $12(\% 80,0)$ & $12(\% 80,0)$ & \\
\hline
\end{tabular}

\#Fisher Exact Chi-Square test

\section{DISCUSSION}

Posterior spine surgery is a very painful procedure for patients on the first postoperative day [6]. Although opioids are an important pillar of postoperative analgesia treatment, high doses given for severe pain control have important side effects such as cognitive impairment, respiratory depression, addiction, constipation, nausea and vomiting [7]. The risk of cardiac and respiratory complications increases in patients without effective pain control. In addition, delay in mobilization due to pain is a separate problem in these patients. Hospital stay is prolonged and chronic pain may occur [8]. The paravertebral muscles and the posterior elements of the spine are innervated by the dorsal roots of the 
spinal nerves. Dorsal roots are separated from the root immediately after the exit of the spinal nerve from the foramen and are distributed in the superficial soft tissue. In the erector spinae plane block, the local anesthetic diffuses in the musculofascial plane and affects the dorsal roots of the spinal nerve at several levels [9]. In addition to systemic analgesic therapies, regional methods also play an important role in multimodal analgesic therapy. Neuraxial blocks, including patient-controlled bolus or continuous infusion of epidural and spinal blocks, are some of the regional methods. It is an important advantage that the ESPB only blocks sensory fibers compared to other peripheral nerve blocks $[10,11]$. ESPB have been successfully used in abdominal surgery, neuropathic pain, pneumothorax and breast implant surgery $[4,12,13]$. In this study we used ESPB for postoperative pain relief after spinal instrumentation surgery. Chin $\mathrm{KJ}$ et al. demonstrated that interfacial blocks provide long-term postoperative pain control and reduce opioid use. 20 $\mathrm{mL}$ of local anesthetic injection has been shown to extend from the infiltration site to 3 or 4 levels caudal [14]. We used $20 \mathrm{~mL}$ bupivacaine injections for each side. Al-Alami et al. conducted a study describing the ultrasound-guided dorsal root block at the T12 and L5 level, but did not present a report containing patient outcome data [15]. We described a c-arm scopy-guided ESPB and reported the outcomes of patients about postoperative pain score, opioid use and its side effects.

\section{CONCLUSION}

In conclusion, on the basis of this retrospective study, it appears that c-arm scopy-guided ESP block, is a safe and effective technique for postoperative pain management, can reduce opioid use and its side effects

\section{REFERENCES}

1. Mathiesen $\mathrm{O}$, Dahl $\mathrm{B}$, Thomsen BA, et al. A comprehensive multimodal pain treatment reduces opioid consumption after multilevel spine surgery. Eur Spine J 2013;22:2089-96.

2. Devin CJ, McGirt MJ. Best evidence in multimodal pain management in spine surgery and means of assessing postoperative pain and functional outcomes. J Clin Neurosci 2015;22:930-8.

3. Bajwa SJ, Haldar R. Pain management following spinal surgeries: An appraisal of the available options. J Craniovertebr Junction Spine 2015;6:105-10.

4. Forero M, Adhikary SD, Lopez H, Tsui C, Chin KJ. The erector spinae plane block: a novel analgesic technique in thoracic neuropathic pain. Reg Anesth Pain Med 2016; 41: 621-7.

5. Chin KJ, Adhikary S, Sarwani N, Forero M. The analgesic efficacy of pre-operative bilateral erector spinae plane (ESP) blocks in patients having ventral hernia repair. Anaesthesia 2017; 72: 452-60.

6. Gerbershagen HJ, Aduckathil S, van Wijck AJ, Peelen LM, Kalkman CJ, Meissner W. Pain intensity on the first day after surgery: a prospective cohort study comparing 179 surgical procedures. Anesthesiology 2013; 118: 934-44.

7. Savarese JJ, Tabler NG Jr. Multimodal analgesia as an alternative to the risks of opioid monotherapy in surgical pain management. J Healthc Risk Manag 2017; 37: 24-30.

8. Thepsoparn M, Sereeyotin J, Pannangpetch P: Effects of combined lower thoracic epidural/general anesthesia on pain control in patients undergoing elective lumbar spine surgery: a randomized controlled trial. Spine (Phila $\mathrm{Pa}$ 1976). 2018, 43:1381-85.

9. Saito $T$, Steinke $H$, Miyaki $T$, et al. Analysis of the posterior ramus of the lumbar spinal nerve: the structure of the posterior ramus of the spinal nerve. Anesthesiology 2013; 118: 88-94.

10. Sinatra RS, Torres J, Bustos AM: Pain management after major orthopaedic surgery: current strategies and new concepts. J Am Acad Orthop Surg. 2002, 10:117-29.

11. Andreae $\mathrm{MH}$, Andreae DA. Regional anaesthesia to prevent chronic pain after surgery: a Cochrane systematic review and meta-analysis. $\mathrm{Br} J$ Anaesth 2013;111:711-20.

12. Bonvicini D, Giacomazzi A, Pizzirani E. Use of the ultrasound-guided erector spinae plane block in breast surgery. Minerva Anestesiol 2017; 83: 1111-2.

13. Ueshima $\mathrm{H}$, Otake $\mathrm{H}$. Erector spinae plane block provides effective pain management during pneumothorax surgery. J Clin Anesth 2017; 40: 74. [CrossRef]

14. Chin KJ, McDonnell JG, Carvalho B, Sharkey A, Pawa A, Gadsden J: Essentials our current understanding: abdominal wall blocks. Reg Anesth Pain Med. 2017, 42:133-83. 10.1097/AAP.0000000000000545

15. Al-Alami A, Abou El Ezz A, Kassab F. Ultrasound guideddorsal ramus nerve block for reduction of postoperative pain in patients undergoing lumbar spine surgery: a case series imaging study. Middle East J Anaesthesiol 2015;23:251-6. 